\title{
Determinants of regulated proteolysis in signal transduction
}

\author{
Sonja Hasenbein, ${ }^{1}$ Melisa Merdanovic, ${ }^{1}$ and Michael Ehrmann ${ }^{1,2,3}$ \\ ${ }^{1}$ Zentrum für Medizinische Biotechnologie, Universität Duisburg-Essen, 45117 Essen, Germany; ${ }^{2}$ School of Biosciences, \\ Cardiff University, Cardiff CF10 3US, United Kingdom
}

Each cell produces thousands of gene products that differ in cellular localization, chemical properties, interactions with other cellular factors, abundance, and half lives. Controlling these events presents an enormous organizational challenge. In recent years, biological qualitycontrol systems were identified addressing this problem. The importance of these systems is underlined by the facts that several quality-control factors are essential for viability and are directly implicated in many severe diseases, such as cancer, cystic fibrosis, amyloid, and autoimmune diseases.

Protein quality control is in charge of the regulation of protein composition by ensuring that individual proteins are synthesized when needed, are properly localized, and are present in the active conformation and in sufficient numbers. To function efficiently, these systems need to be able to examine the surface of proteins, diagnose defects, signal problems, decide to repair or destroy substrates, and re-establish the steady state. As cellular compartments have a specific repertoire of proteins and are separated from each other by semipermeable membranes, each of them has dedicated quality-control systems. The first protein quality-control system identified was the cytoplasmic heat-shock regulon in Escherichia coli that senses and responds to misfolded proteins that accumulate at elevated temperatures (Arsene et al. 2000). Its key elements are several protein repair factors including the molecular chaperones Hsp60 and Hsp70, proteases such as Hsp100, and dedicated transcription factors that control the time and level of the response (Bukau and Horwich 1998; Bukau et al. 2006). Subsequently, an additional heat-shock system driven by the alternative $\sigma$ factor $\mathrm{E}$ (RpoE) has been identified (Erickson and Gross 1989). This system is an important feature of the cell envelope of Gram-negative bacteria, consisting of the periplasm and the outer membrane. Here, $\sigma^{\mathrm{E}}$ controls protein composition and function including the biogenesis of outer-membrane proteins (Raivio and Silhavy 2001; Alba and Gross 2004; Ruiz and Silhavy 2005).

\footnotetext{
${ }^{3}$ Corresponding author.

E-MAIL michael.ehrmann@uni-due.de or ehrmann@cf.ac.uk; FAX 49201-183-3315.

Article is online at http://www.genesdev.org/cgi/doi/10.1101/gad.1507807.
}

It is of interest that in contrast to the cytoplasm, cell envelope protein quality control functions in the absence of ATP. Also, bacterial cells do not rely on only one system. The well-studied two-component regulatory system Cpx and the less well-understood Bae system provide an additional level of control in the regulation of stress promoters (Raivio and Silhavy 2001). In this issue of Genes \& Development, Chaba et al. (2007) present new insights into the proteolytic cascade that controls expression of $\sigma^{\mathrm{E}}$-mediated envelope stress response in $E$. coli

$\sigma^{\mathrm{E}}$ is responsible for the differential activity of dozens of promoters in response to various events that require elevated levels of protein-folding factors and proteases. It influences the production of lipopolysaccharide and the integrity of the outer membrane, and it contributes significantly to the survival of pathogens in a host (Raivio 2005; Rhodius et al. 2006). Events that induce $\sigma^{\mathrm{E}}$ include stress conditions that interfere with protein folding, such as elevated temperatures or ethanol in the medium, but also the overproduction of outer-membrane proteins or mutations that cause folding problems (Mecsas et al. 1993).

\section{The components involved in signal sensing and signal transduction}

The problem of misfolded or mislocalized proteins in the bacterial cell envelope must be communicated to the cytoplasm where the response is initiated by differential gene expression. Successful signal transduction depends on sensing and the actual mechanism of transduction across the cytoplasmic membrane as well as activation of the alternative $\sigma$ factor $E$. This process requires the participation of several proteins (Fig. 1). Under latent conditions, $\sigma^{\mathrm{E}}$ is kept inactive by interaction with the anti- $\sigma$ factor RseA. RseA is an integral cytoplasmic membrane protein. It is composed of a cytoplasmic domain that binds $\sigma^{\mathrm{E}}$, one transmembrane segment, and a periplasmic domain. When misfolded proteins appear in the periplasm, they are sensed by the serine protease DegS, a membrane protein with a periplasmic catalytic domain. This protease is completely inactive in the absence of stress signals. After the binding of stress pep- 


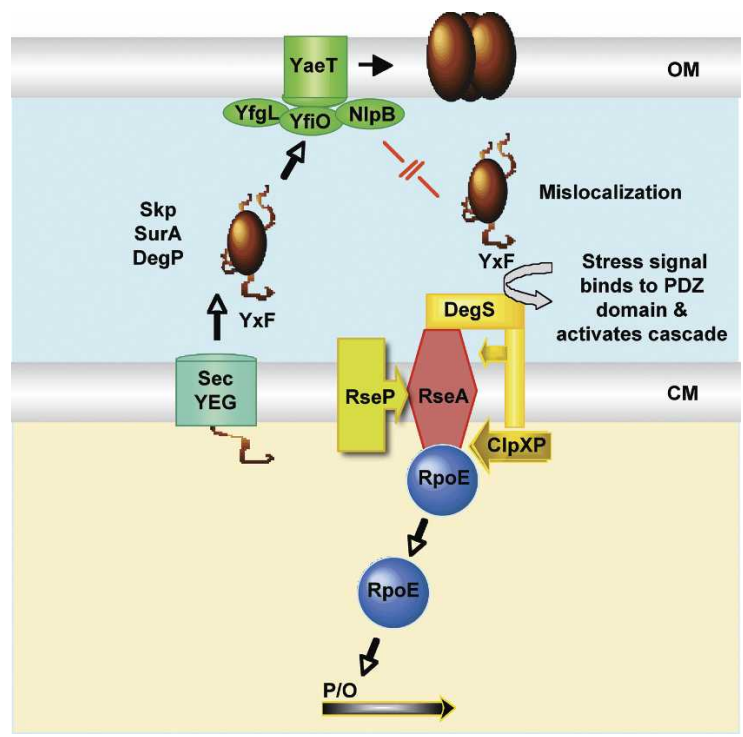

Figure 1. Schematic model of the $\sigma^{\mathrm{E}}$ pathway. Outer-membrane proteins (shown in brown) are secreted into the periplasm via the Sec system. Initially, periplasmic folding factors Skp, SurA, and DegP prevent aggregation of outer-membrane proteins prior to membrane insertion and might be involved in their transport to the NlpB-YfgL-YfiO-YaeT protein complex to facilitate insertion into the outer membrane. Outer-membrane proteins that are mislocalized to the periplasm interact via their $\mathrm{C}$ termini with DegS. This interaction causes activation of DegS, which initiates a proteolytic cascade leading to the degradation of RseA. Proteolysis of RseA occurs in three sequential steps. DegS cleaves RseA in the periplasm, the RIP protease RseP within the membrane, and ClpXP in the cytoplasm. Subsequently, $\sigma^{\mathrm{E}}(\mathrm{RpoE})$ is released and activates stress promoters (see the text for further details). (OM) Outer membrane; (CM) cytoplasmic membrane.

tides, DegS is activated and cleaves RseA near the cytoplasmic membrane. Two subsequent proteolytic events lead to further processing of the remaining RseA fragment within the cytoplasmic membrane via the RIP (regulated intramembrane proteolysis) protease RseP (YaeL) and the final destruction of the cytoplasmic domain of RseA by various cytoplasmic proteases including ClpXP and Lon. Once RseA has been degraded, $\sigma^{\mathrm{E}}$ is released to interact with RNA polymerase to differentially regulate its target promoters. Beside these core elements, accessory factors such as RseB are involved in additional control of the signal transduction pathway (De Las Penas et al. 1997b; Grigorova et al. 2004). Interestingly, $\operatorname{deg} S$ and $r s e P$ are essential for viability because $\sigma^{\mathrm{E}}$ function is essential (De Las Penas et al. 1997a).

The mechanism of signal sensing and signal transduction

The signal

One signal activating the $\sigma^{\mathrm{E}}$ response has been characterized in detail. This signal is a short peptide sequence corresponding to the $\mathrm{C}$ terminus of the outer-membrane protein $\mathrm{C}(\mathrm{OmpC})$. OmpC belongs to the widely conserved family of porins that mediate the exchange of solutes between the medium and the periplasm (Nikaido 2003). Porins form homotrimers in the outer membrane. In each monomer, even numbers of consecutively arranged $\beta$-strands form the $\beta$-barrels that are embedded in the membrane. In addition, the borders of $\beta$-strands contain aromatic residues. Their side chains point to the polar head groups of lipids, suggesting an anchoring function in the membrane. Interestingly, the last residue of the $\mathrm{C}$-terminal $\beta$-strand is in most cases an aromatic residue.

As is the case for many $\beta$-barrel proteins, the $C$ terminus of OmpC is part of the last $\beta$-strand (Basle et al. 2006). Therefore, once the protein has reached its final destination, its $\mathrm{C}$ terminus is hidden in the membrane (Fig. 2). If misfolding or overproduction occur, OmpC mislocalizes to the periplasm, exposing its $\mathrm{C}$ terminus. This C-terminal peptide sequence is then sensed by DegS, triggering the $\sigma^{\mathrm{E}}$ response (Walsh et al. 2003). In addition, different amino acids in the -1 position of the stress peptide cause different levels of DegS activity (Wilken et al. 2004). This result suggests that DegS activity can be fine-tuned, leading to graded $\sigma^{\mathrm{E}}$ response. There may be several reasons why the $\mathrm{C}$ terminus of outer-membrane proteins serves as a stress signal. First, outer-membrane proteins can be highly abundant. For example, Maltoporin and OmpA are produced in 100,000 copies per cell (Koebnik et al. 2000). Misfolded outermembrane proteins can therefore present a considerable burden for cells, as is exemplified by the lethal effects of

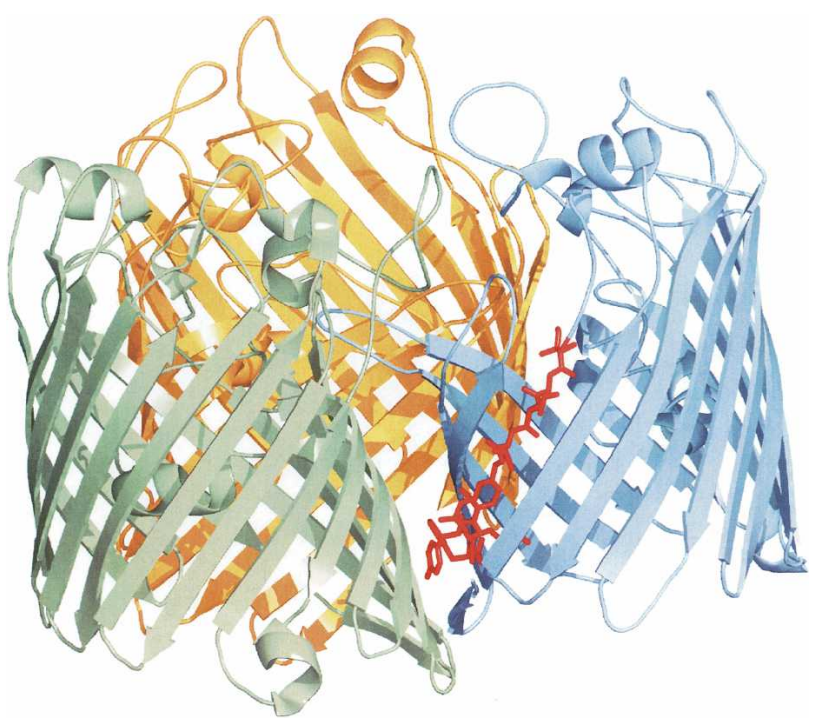

Figure 2. Three-dimensional structure of trimeric osmoporin $\mathrm{OmpC}$ of E. coli. Individual monomers are shown in green, blue, and orange. In one of the subunits, the C-terminal peptide $\mathrm{I}_{337}$ VALGLVYQF $_{346}$ serving as a signal for cell envelope-folding stress and the $\sigma^{\mathrm{E}}$ response is highlighted in red (stick representation). In properly folded OmpC, this peptide is buried in the interface between two subunits and is only accessible as a consequence of protein misfolding and mislocalization to the periplasm. The PDB code for the OmpC structure is $2 \mathrm{~J} 1 \mathrm{~N}$. 
certain ompC and $o m p F$ mutations that cause misfolding (Misra et al. 2000; CastilloKeller and Misra 2003). In addition, rseP becomes nonessential in the absence of OmpC and OmpA (Douchin et al. 2006). Second, since the very $C$ termini of outer-membrane proteins are often part of the final $\beta$-strand and thus important for folding and $\beta$-barrel formation, this sequence is well-suited to serve as a stress signal. Third, PDZ domains preferentially bind $\mathrm{C}$ termini of other proteins (Saras and Heldin 1996). Accordingly, sequences that activate DegS carry a YxF (e.g., YQF, YYF, and YWF) motif corresponding to the $\mathrm{C}$ terminus of many outer-membrane proteins (Walsh et al. 2003).

\section{The proteolytic cascade}

\section{Step 1: initial processing of RseA by DegS}

As shown by the accompanying paper by Chaba et al. (2007), confirming earlier results (Ades et al. 2003), DegS performs the rate-limiting reaction in RseA processing and $\sigma^{\mathrm{E}}$ activation. In vivo pulse-chase proteolysis experiments determining the kinetics of the first step of RseA degradation revealed that $T_{1 / 2}$ is $\sim 1 \mathrm{~min}$, leading to an intermediate product that is subsequently further processed by RseP.

DegS belongs to the HtrA family of serine proteases that is defined by a variable $\mathrm{N}$-terminal domain, a serine protease domain resembling trypsin, and one or two Cterminal PDZ domains (Clausen et al. 2002). PDZ domains are protein interaction modules of $\sim 100$ amino acids that preferentially bind $\mathrm{C}$ termini of other proteins in a $\beta$-augmentation process (Saras and Heldin 1996). Members of the HtrA family differ from the well-studied classical serine proteases by their ability to undergo a reversible switch between the active and inactive conformations. This switch is brought about by a mechanism termed reversible zymogen activation. It allows these proteases to quickly respond to environmental signals (Ehrmann and Clausen 2004). In addition, DegS is an extremely robust protein, tolerating $5 \mathrm{M} \mathrm{NaCl}$, treatment with $6 \mathrm{M}$ guanidinium hydrochloride, and temperatures between $10^{\circ} \mathrm{C}$ and $50^{\circ} \mathrm{C}$ (S. Hasenbein and M.Ehrmann, unpubl.). As a membrane protein, DegS is well positioned to cleave the anti- $\sigma$ factor RseA near the periplasmic face of the cytoplasmic membrane. Crystal structures of trimeric DegS revealed that it is present in an enzymatically inactive conformation in the absence of stress signals (Wilken et al. 2004). The inactivity of DegS is caused by an improperly positioned catalytic triad, a misformed oxyanion hole, occluded substrate specificity pockets, and the absence of structures involved in main-chain binding of substrates. Upon binding of stress signals (peptides resembling the $\mathrm{C}$ terminus of outer-membrane proteins) to the PDZ domains, loop 3 of the protease domain reorients its position to bind to the -1 residue of the stress-peptide (e.g., Q of YQF or W of YWF). Reorientation of loop 3 causes other loops of the protease domain including the active site loops L1, L2, and LD to undergo conformational changes that ul- timately lead to the formation of the active conformation. Activated DegS initiates proteolytic inactivation of RseA by cleaving between Val148 and Ser149 (Walsh et al. 2003).

\section{Step 2: Rse $A^{1-148}$ is further processed by RseP}

The work of Chaba et al. (2007) indicates that subsequent processing of the RseA fragment 1-148 within the membrane occurs very rapidly with a $T_{1 / 2}$ of $\sim 20 \mathrm{sec}$. This reaction leads to the second intermediate product, RseA $^{1-108}$ (Akiyama et al. 2004).

The metalloprotease RseP belongs to two protease superfamilies. One is the diverse family of RIP proteases that catalyze the processing of membrane-spanning segments within the lipid bilayer. Prominent family members include $\gamma$-secretase, rhomboid, site 2 protease, and signal-peptide peptidase (Urban and Freeman 2002). RseP belongs also to the PDZ proteases including the HtrA family and thus DegS. In contrast to DegS, which has a C-terminal PDZ domain, the PDZ domain of RseP is sandwiched between the two N- and C-terminal membrane-spanning segments and is thus located in the middle of the protein. The function of the PDZ domain might be regulatory and thus similar to that of DegS, as deletion analyses indicated that PDZ-less RseP exhibits elevated proteolytic activity and altered substrate specificity. The latter leads to cleavage of full-length RseA that is normally not processed by RseP (Kanehara et al. 2003; Bohn et al. 2004).

The general functional mechanism of RIP proteases is not yet well understood. However, a recent crystallographic analysis of the rhomboid GlpG from E. coli provides a first structural model (Wang et al. 2006). Even though GlpG is a serine protease and has six transmembrane segments, its structure exhibits features that might be common to all RIP proteases. These features include the presence of a cavity within the membrane containing the active site. A V-shaped gap in the cavity provides enough space to allow lateral entry of a membrane-spanning substrate. In addition, several water molecules that are important for catalysis are bound by the active site. It is also postulated that a large loop connecting transmembrane helices 1 and 2 functions as a gate. The PDZ domain of RseP might carry out a similar function.

Step 3: Rse $A^{1-108}$ is degraded by cytoplasmic proteases

Even though DegS and RseP cut RseA in half, the remaining N-terminal part of RseA still binds the $\sigma$ factor very tightly with pM affinity (Chaba et al. 2007). Crystallographic analysis of the RseA $-\sigma^{\mathrm{E}}$ complex revealed that this tight binding is the consequence of multiple interactions between the two proteins (Campbell et al. 2003). Therefore, it is believed that $\sigma^{\mathrm{E}}$ is unable to spontaneously dissociate from the cytoplasmic RseA fragment. In agreement with this model, several ATP-dependent cytoplasmic proteases have been identified to 
cleave RseA. The final step of RseA degradation occurs again rapidly - in the range of $20 \mathrm{sec}$-allowing $\sigma^{\mathrm{E}}$ to be released and to interact with RNA polymerase. Mutational analyses and in vitro protease assays revealed that ClpXP and Lon are the major degrading factors, while slower reactions can be carried out by other proteases (Chaba et al. 2007).

\section{Step 4: $\sigma^{E}$ modulates expression of target promoters}

Following its release from the anti- $\sigma$ factor RseA, $\sigma^{\mathrm{E}}$ activates transcription of its target promoters. Transcriptional profiling has identified 50 transcriptional units that are differentially expressed (Rhodius et al. 2006). This analysis indicates that ClpXP and Lon are members of the $\sigma^{\mathrm{E}}$ regulon. Their up-regulation following folding stress supports rapid degradation of the final RseA fragment. The levels of other members of the proteolytic cascade, DegS and RseP, are not, however, regulated by $\sigma^{\mathrm{E}}$. Several lines of evidence suggest that the folding and insertion of outer-membrane proteins receives special attention by the protein quality-control machinery of the cell envelope. First, the recently identified members of the protein insertion machinery of the outer membrane-NlpB, YaeT, YfgL, and YfiO (for review, see Ruiz et al. 2006)-are also up-regulated by $\sigma^{\mathrm{E}}$. Second, the production of other proteins (Skp, SurA, and DegP) previously implicated in outer-membrane protein biogenesis (Mogensen and Otzen 2005) is also at least in part $\sigma^{\mathrm{E}}$ dependent. Third, negative regulation of six genes encoding outer-membrane proteins, including OmpC (Guillier et al. 2006; Johansen et al. 2006; Rhodius et al. 2006), lowers the burden.

\section{Conclusions and implications}

The main players of the $\sigma^{\mathrm{E}}$ signal transduction cascade are identified, the mechanism of signal sensing and proteolytic activation of the rate-limiting factor DegS are understood at the atomic level, and the kinetics of the entire proteolytic cascade are revealed (Fig. 1). The complete process from signal sensing to $\sigma^{\mathrm{E}}$ release takes no more than $2 \mathrm{~min}$, allowing a rapid response to proteinfolding or mislocalization problems in the cell envelope.

These data also reveal general features of regulated proteolysis in signal transduction. In the latent state, a negative regulator (e.g., an anti- $\sigma$ factor) represses stress response. Short peptide sequences of protein quality-control substrates that are inaccessible under normal conditions serve as stress signals. After exposure, they are sensed by the first protease involved in destruction of the negative regulator. The activity of this protease is tightly controlled and rate limiting. Further processing of the negative regulator via a RIP protease occurs within the membrane. This second cleavage requires initial processing, as the RIP protease is unable to degrade a full-length substrate. A third proteolytic event completes the removal of the negative regulator and activates stress response. In this regulated proteolysis pathway, each player adheres to its specific role and several modes of regulation of activity mediate a graded response.

These insights have wide implications. The $\sigma^{\mathrm{E}}$ pathway may serve as a role model for other signal transduction systems that involve regulated proteolysis and for computational simulations in systems biology. Members of the $\sigma^{\mathrm{E}}$ regulon can be modeled into the network of protein-folding factors and proteases involved in the control of protein composition in the cell envelope. In addition, a detailed understanding of stress response will enable practical applications including the use of DegS, RseP, and $\sigma^{\mathrm{E}}$ as drug targets and the exploration of the use of $\sigma^{\mathrm{E}}$-regulated gene products in protein production.

\section{Acknowledgments}

We thank Deutsche Forschungsgemeinschaft and the British Biotechnology and Biological Sciences Research Council for support.

\section{References}

Ades, S., Grigorova, I., and Gross, C. 2003. Regulation of the alternative $\sigma$ factor $\sigma^{\mathrm{E}}$ during initiation, adaptation, and shutoff of the extracytoplasmic heat shock response in Escherichia coli. J. Bacteriol. 185: 2512-2519.

Akiyama, Y., Kanehara, K., and Ito, K. 2004. RseP (YaeL), an Escherichia coli RIP protease, cleaves transmembrane sequences. EMBO J. 23: 4434-4442.

Alba, B.M. and Gross, C.A. 2004. Regulation of the Escherichia coli $\sigma$-dependent envelope stress response. Mol. Microbiol. 52: 613-619.

Arsene, F., Tomoyasu, T., and Bukau, B. 2000. The heat shock response of Escherichia coli. Int. J. Food Microbiol. 55: 3-9.

Basle, A., Rummel, G., Storici, P., Rosenbusch, J., and Schirmer, T. 2006. Crystal structure of osmoporin OmpC from E. coli at 2.0 Å. J. Mol. Biol. 362: 933-942.

Bohn, C., Collier, J., and Bouloc, P. 2004. Dispensable PDZ domain of Escherichia coli YaeL essential protease. Mol. Microbiol. 52: 427-435.

Bukau, B. and Horwich, A. 1998. The Hsp70 and Hsp60 chaperone machines. Cell 92: 351-366.

Bukau, B., Weissman, J., and Horwich, A. 2006. Molecular chaperones and protein quality control. Cell 125: 443-451.

Campbell, E.A., Tupy, J.L., Gruber, T.M., Wang, S., Sharp, M.M., Gross, C.A., and Darst, S.A. 2003. Crystal structure of Escherichia coli $\sigma^{\mathrm{E}}$ with the cytoplasmic domain of its anti- $\sigma$ RseA. Mol. Cell 11: 1067-1078.

CastilloKeller, M. and Misra, R. 2003. Protease-deficient DegP suppresses lethal effects of a mutant OmpC protein by its capture. J. Bacteriol. 185: 148-154.

Chaba, R., Grigorova, I.L., Flynn, J.M., Baker, T.A., and Gross, C.A. 2007. Design principles of the proteolytic cascade governing the $\sigma^{\mathrm{E}}$-mediated envelope stress response in Escherichia coli: Keys to graded, buffered and rapid signal transduction. Genes \& Dev. (this issue).

Clausen, T., Southan, C., and Ehrmann, M. 2002. The HtrA family of proteases. Implications for protein composition and cell fate. Mol. Cell 10: 443-455.

De Las Penas, A., Connolly, L., and Gross, C.A. 1997a. $\sigma^{\mathrm{E}}$ is an essential $\sigma$ factor in Escherichia coli. J. Bacteriol. 179: 68626864.

De Las Penas, A., Connolly, L., and Gross, C.A. 1997b. The 
$\sigma^{\mathrm{E}}$-mediated response to extracytoplasmic stress in Escherichia coli is transduced by RseA and RseB, two negative regulators of $\sigma^{\mathrm{E}}$. Mol. Microbiol. 24: 373-385.

Douchin, V., Bohn, C., and Bouloc, P. 2006. Down-regulation of porins by a small RNA bypasses the essentiality of the regulated intramembrane proteolysis protease RseP in Escherichia coli. J. Biol. Chem. 281: 12253-12259.

Ehrmann, M. and Clausen, T. 2004. Proteolysis as a regulatory mechanism. Annu. Rev. Genet. 38: 709-724.

Erickson, J. and Gross, C. 1989. Identification of the $\sigma^{\mathrm{E}}$ subunit of Escherichia coli RNA polymerase: A second alternate ofactor involved in high-temperature gene expression. Genes \& Dev. 9: 1462-1471.

Grigorova, I.L., Chaba, R., Zhong, H.J., Alba, B.M., Rhodius, V., Herman, C., and Gross, C.A. 2004. Fine-tuning of the Escherichia coli $\sigma^{\mathrm{E}}$ envelope stress response relies on multiple mechanisms to inhibit signal-independent proteolysis of the transmembrane anti- $\sigma$ factor, RseA. Genes \& Dev. 18: 26862697.

Guillier, M., Gottesman, S., and Storz, G. 2006. Modulating the outer membrane with small RNAs. Genes \& Dev. 20: 2338 2348.

Johansen, J., Rasmussen, A., Overgaard, M., and Valentin-Hansen, P. 2006. Conserved small non-coding RNAs that belong to the $\sigma^{\mathrm{E}}$ regulon: Role in down-regulation of outer membrane proteins. J. Mol. Biol. 364: 1-8.

Kanehara, K., Ito, K., and Akiyama, Y. 2003. YaeL proteolysis of RseA is controlled by the PDZ domain of YaeL and a Glnrich region of RseA. EMBO J. 22: 6389-6398.

Koebnik, R., Locher, K.P., and Van Gelder, P. 2000. Structure and function of bacterial outer membrane proteins: Barrels in a nutshell. Mol. Microbiol. 37: 239-253.

Mecsas, J., Rouviere, P.E., Erickson, J.W., Donohue, T.J., and Gross, C.A. 1993. The activity of $\sigma^{\mathrm{E}}$, an Escherichia coli heat-inducible $\sigma$-factor, is modulated by expression of outer membrane proteins. Genes \& Dev. 7: 2618-2628.

Misra, R., CastilloKeller, M., and Deng, M. 2000. Overexpression of protease-deficient $\operatorname{DegP}(\mathrm{S} 210 \mathrm{~A})$ rescues the lethal phenotype of Escherichia coli OmpF assembly mutants in a degP background. J. Bacteriol. 182: 4882-4888.

Mogensen, J. and Otzen, D. 2005. Interactions between folding factors and bacterial outer membrane proteins. Mol. Microbiol. 57: 326-346.

Nikaido, H. 2003. Molecular basis of bacterial outer membrane permeability revisited. Microbiol. Mol. Biol. Rev. 67: 593656.

Raivio, T. 2005. Envelope stress responses and Gram-negative bacterial pathogenesis. Mol. Microbiol. 56: 1119-1128.

Raivio, T.L. and Silhavy, T.J. 2001. Periplasmic stress and ECF $\sigma$ factors. Annu. Rev. Microbiol. 55: 591-624.

Rhodius, V., Suh, W., Nonaka, G., West, J., and Gross, C. 2006. Conserved and variable functions of the $\sigma^{\mathrm{E}}$ stress response in related genomes. PLoS Biol. 4: e2.

Ruiz, N. and Silhavy, T. 2005. Sensing external stress: Watchdogs of the Escherichia coli cell envelope. Curr. Opin. Microbiol. 8: 122-126.

Ruiz, N., Kahne, D., and Silhavy, T. 2006. Advances in understanding bacterial outer-membrane biogenesis. Nat. Rev. Microbiol. 4: 57-66.

Saras, J. and Heldin, C.H. 1996. PDZ domains bind carboxyterminal sequences of target proteins. Trends Biochem. Sci. 21: $455-458$

Urban, S. and Freeman, M. 2002. Intramembrane proteolysis controls diverse signalling pathways throughout evolution. Curr. Opin. Genet. Dev. 12: 512-518.

Walsh, N.P., Alba, B.M., Bose, B., Gross, C.A., and Sauer, R.T.
2003. OMP peptide signals initiate the envelope-stress response by activating DegS protease via relief of inhibition mediated by its PDZ domain. Cell 113: 61-71.

Wang, Y., Zhang, Y., and Ha, Y. 2006. Crystal structure of a rhomboid family intramembrane protease. Nature 444: 179180

Wilken, C., Kitzing, K., Kurzbauer, R., Ehrmann, M., and Clausen, T. 2004. Crystal structure of the DegS stress sensor: How a PDZ domain recognizes misfolded protein and activates a protease domain. Cell 117: 483-494. 


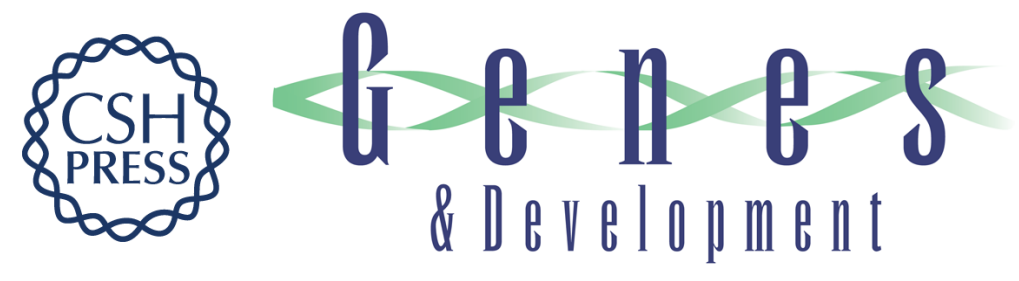

\section{Determinants of regulated proteolysis in signal transduction}

Sonja Hasenbein, Melisa Merdanovic and Michael Ehrmann

Genes Dev. 2007, 21:

Access the most recent version at doi:10.1101/gad.1507807

Related Content Design principles of the proteolytic cascade governing the ÃE-mediated envelope stress response in Escherichia coli: keys to graded, buffered, and rapid signal transduction

Rachna Chaba, Irina L. Grigorova, Julia M. Flynn, et al.

Genes Dev. January , 2007 21: 124-136

References This article cites 35 articles, 11 of which can be accessed free at: http://genesdev.cshlp.org/content/21/1/6.full.html\#ref-list-1

Articles cited in:

http://genesdev.cshlp.org/content/21/1/6.full.html\#related-urls

\section{License}

Email Alerting

Service

Receive free email alerts when new articles cite this article - sign up in the box at the top right corner of the article or click here.

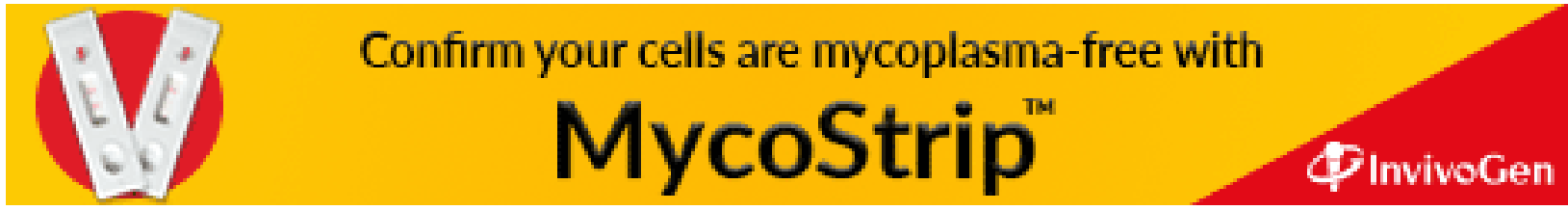

